\title{
Cerebral midline structures in bimanual coordination
}

Received: 20 September 1998 / Accepted: 24 February 1999

\begin{abstract}
In six healthy right-handed volunteers, we compared the cerebral activation pattern related to unimanual right- and left-hand movements and to bimanual in-phase and anti-phase movements using functional magnetic resonance imaging (fMRI). Internally paced unimanual finger-to-thumb opposition movements led to a strong contralateral activation of primary sensorimotor areas in all six subjects. Midline activity was lateralized to the left side during right-hand movements, but to both sides during left-hand movements. Activity patterns of bimanual in-phase movements resembled the combined activity patterns of the two unimanual conditions: right and left hemispheric activations of the primary sensorimotor cortices and predominantly left-sided medial frontal activity. In contrast, during anti-phase movements, we observed a clear increase in activity, in both right and left frontal midline areas and in right hemispheric, mainly dorsolateral premotor areas compared to in-phase movements. These results indicate that frontal midline activity is not specific for bimanual movements per se. It can already be involved during simple unimanual movements but becomes progressively more involved during more complex aspects of movement control.
\end{abstract}

Key words Bimanual movements · Mesial frontal cortex $\cdot$ Supplementary motor area (SMA) - Cingulate motor areas (CMA) · Functional magnetic resonance imaging (fMRI)

\section{Introduction}

Medial frontal areas are often seen as an important structure for bimanual coordination. The close relationship of

K.M. Stephan $(-$ F. Binkofski · R.J. Seitz · H.-J. Freund

Neurologische Universitätsklinik,

Heinrich-Heine Universität Düsseldorf, Moorenstraße 5,

D-40225 Düsseldorf, Germany,

Tel: +49 211811 7044, Fax: +49 2118118469

S. Posse

Institut für Medizin, Forschungszentrum Jülich, Jülich, Germany the medial frontal cortex with bilateral movements is supported by anatomical, electrophysiological and lesion data in the monkey and in humans. Anatomically, there are strong interhemispheric connections between the two supplementary motor areas (SMAs) including distal representations of arm and hand (Rouiller et al. 1994) and strong bilateral SMA projections to the basal ganglia. Neuronal activity within the SMA was associated both with contralateral and ipsilateral arm movements (Brinkman and Porter 1979; Tanji et al. 1988). Furthermore, bimanual movement sequences were impaired by lesions in the frontomesial cortex including the SMA and anterior cingulate (e.g., Travis 1955; Brinkman 1984; Laplane et al. 1977).

On the other hand, kinematic analysis of the effect of well-defined lesions of the SMA showed a surprising stability and precision of the final interaction of the two hands at the target (Kazennikov et al. 1994, 1998; Wiesendanger et al. 1996). This preservation of "goal invariance" in conjunction with neuronal recording data (Kazennikov et al. 1999) led the authors to conclude that the SMA is clearly engaged in bimanual tasks, but does not represent the bimanual command structure.

We recently investigated two patients with cingulate lesions and a well-defined bimanual coordination deficit. The nature of their deficit and the results of a functional magnetic resonance imaging (fMRI) activation study for bimanual in-phase and anti-phase movements highlighted the importance of medial frontal and especially cingulate motor areas for temporal aspects of bimanual coordination (Stephan et al. 1999). In the present paper, we investigate the specificity of the observed activation patterns for bimanual control: we compare the degree of cortical activity during simple unimanual right and left finger-thumb opposition movements with that observed during the bimanual in-phase and anti-phase movements. Our results suggest that the degree and the site of activity within medial frontal areas are more related to the temporal nature of the task and its degree of complexity than to the simple fact that movements are performed by either one or two hands. 


\section{Materials and methods}

We investigated six right-handed healthy subjects (mean age 32 years) without a history or signs of a neurological or psychiatric illness and without any structural lesion on cranial MRI. The study was approved by the local ethics committee and all subjects gave informed consent.

The healthy volunteers performed four different sets of movements: unimanual index finger-thumb opposition movements with the right hand (A), the same movements with the left hand (B), bimanual finger-thumb opposition movements with both hands inphase (C) and with both hands anti-phase (D). To avoid a systematic influence of the degree of activation due to the different movement rates, we tried to ensure that the subjects performed their movements at similar rates. They were externally paced before performance of each task at $1 \mathrm{~Hz}$ while lying in the MR scanner in order to familiarize themselves with the pace of movements. At the beginning of the fMRI sequences, they started anew to perform the task. Subjects tried to stick to the frequency of movements experienced just before, but during MR scanning there was no external pacing.

Functional MRI data were obtained with a Siemens Vision system (1.5 T) and echoplanar sequences (TR $3 \mathrm{~s}$, TE $66 \mathrm{~ms}, 90^{\circ}$ ) utilizing the "bold" contrast. Ten consecutive slices of $4 \mathrm{~mm}$ thickness adjusted to the AC-PC line and positioned above the corpus callosum were acquired. Voxel size was $3 \times 3 \times 4 \mathrm{~mm}$. Five periods of $15 \mathrm{~s}$ rest were each followed by a period of $15 \mathrm{~s}$ activation.

We performed both a group analysis for all six volunteers and an analysis of individual data sets. For both methods successive functional imaging data were realigned for movement correction. After coregistration of the data sets with the individual structural MRIs, the realigned data were transformed into modified "Talairach space" (Talairach and Tournoux 1988) as defined by the standard brain of the Montreal Neurological Institute (MNI). After smoothing with a filter of $8 \mathrm{~mm}$ width, task-rest comparisons were calculated and statistical analysis performed [Statistical Parametric Mapping Program 1996 (SPM '96), Wellcome Department of Cognitive Neurology, Institute of Neurology, London; Friston et al. 1995a, 1995b]. For the group analysis, we used a significance level of $P<0.001$ and for comparisons between conditions (antiphase versus in-phase) a significance level of $P<0.01$ in the identified anatomical areas. Statistical analysis for individual subjects was performed using a significance level of $P<0.001$ for each individual voxel and an extent threshold of $P<0.05$. Comparisons between conditions (anti-phase versus in-phase) were again performed at a significance level of $P<0.01$ in the identified anatomical areas. Statistically significant $t$-maps were then superimposed on the subjects' own MRIs for individual results or on a standard MRI oriented in modified Talairach space (MNI space) for the group results.

Finally, to allow a more detailed comparison of the activation patterns in medial wall areas between the different conditions, we performed a modified region of interest analysis. We defined three distinct areas for both hemispheres (Fig. 1): (1) medial areas just above the cingulate sulcus or - if present - between a paracingulate sulcus and the cingulate sulcus, (2) cingulate areas in the depth of the cingulate sulcus with maxima of activity located at least $9 \mathrm{~mm}$ lateral from the midline (cingulate motor areas) and (3) dorsal medial wall areas (SMA). SPM analysis was performed on the same individual data sets as before; they were, however, smoothed at 4-mm filter width to enhance the signal-to-noise ratio. For each of the three defined areas we compared the number of subjects who showed statistically significant peaks of activation $(P<0.01$, uncorrected) in the SPM $t$-maps in each of the four conditions. Each significant activation was marked with an " $x$ " in Table 3 , leading to a maximum number of six " $x$ "s in every cell of the table. To facilitate comparison between conditions and anatomical areas, we have adapted the size of each " $x$ " to the level of significance ( $t$-values) of each individual activation peak. A small " $x$ " symbolizes a $t$-value of $2.33 \leq t<3.6$, a medium-sized " $x$ " indicates a $t$-value of $3.6 \leq t<5.0$ and a large " $X$ " a $t$-value of $t \geq 5.0$.

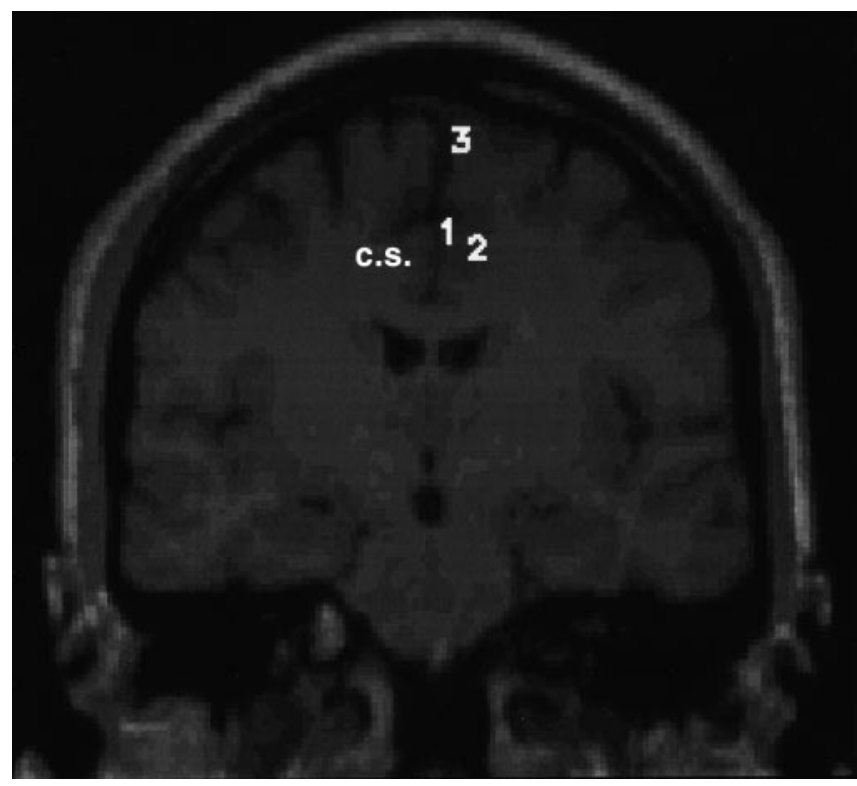

Fig. 1 Schematic illustration showing the location of the areas investigated in the modified region of interest analysis ( 1 ventral motor areas close to the mesial wall, 2 CMA in the depth of the c.s., 3 supplementary motor area, c.s. cingulate sulcus)

\section{Results}

\section{Group results}

The group result showed a strong activation of contralateral primary sensorimotor areas during unimanual and bimanual movements (Fig. 2) without any obvious difference between right and left hemispheres. During unimanual movements regions of the mesial frontal cortex including SMA and cingulate areas became only activated during right-hand movements (12 activated voxels), not during left-hand movements. During bimanual movements the number of activated voxels in the mesial frontal cortex was greater during anti-phase (24 activated voxels) than during in-phase (5 activated voxels) movements. The focus of activation of the mesial frontal cortex can be allocated to the walls of the cingulate sulcus and its opening on the mesial surface mainly on the left side (Fig. 2). A direct comparison between the anti-phase and the in-phase condition showed no significant activity. At subthreshold levels, a small activation was seen at the opening of the cingulate sulcus on the left side.

\section{Single subjects}

Single-subject results confirmed and enhanced the group results: during unimanual finger-thumb opposition movements activations of the contralateral primary sensorimotor and adjacent premotor areas did not show a significant difference between right and left side as measured by the number of activated voxels (Table 1). For both the right-hand and the left-hand condition, the adja- 
A
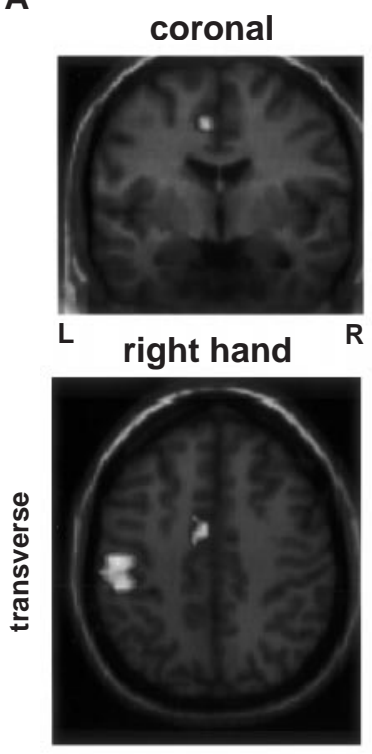

B
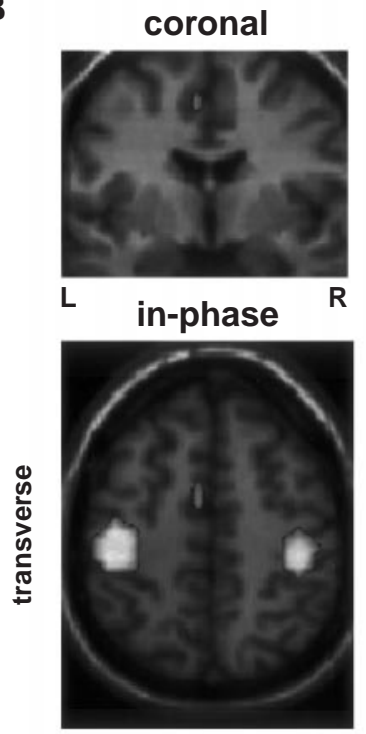

coronal

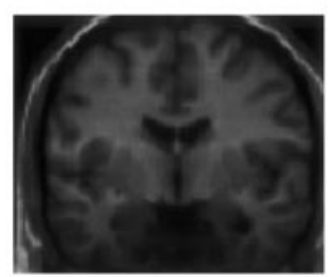

left hand

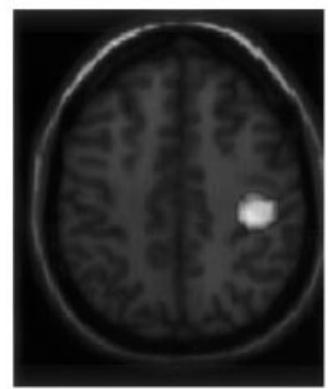

coronal

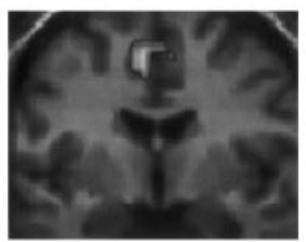

anti-phase

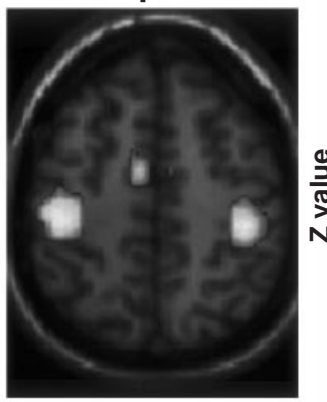

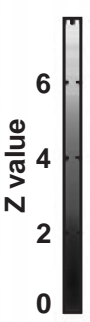

Fig. 2A,B Statistically significant signal increases obtained with fMRI in a group of six right-handed volunteers performing a unimanual and bimanual index-finger thumb opposition task. Unimanual right- and left-hand movements are displayed in the two upper panels, bimanual in-phase and anti-phase movements in the lower panels. Significant voxels $(P<0.01)$ are superimposed on a standard MRI in MNI space (for details see text) ( $L$ left, $R$ right)

Table 1 Activity in lateral hemispheric areas in individual subjects. Number of significant voxels in primary sensorimotor, parietal and premotor areas for all six individual subjects during active conditions versus rest. Statistical significance level: $P<0.001$; corrected for spatial extent: $P<0.05$

\begin{tabular}{|c|c|c|c|c|c|c|c|c|}
\hline \multirow[t]{2}{*}{ Subject } & \multicolumn{2}{|c|}{ Right hand } & \multicolumn{2}{|c|}{ Left hand } & \multicolumn{2}{|l|}{ In-phase } & \multicolumn{2}{|c|}{ Anti-phase } \\
\hline & R. hem. & L. hem. & R. hem. & L. hem. & R. hem. & L. hem. & R. hem. & L. hem. \\
\hline 1 & 57 & 269 & 274 & - & 213 & 218 & 379 & 273 \\
\hline 2 & 45 & 295 & 301 & - & 304 & 254 & 295 & 240 \\
\hline 3 & - & 120 & 170 & - & 181 & 164 & 343 & 171 \\
\hline 4 & - & 92 & 85 & - & 83 & 119 & 104 & 148 \\
\hline 5 & - & 86 & 147 & - & 138 & 125 & 196 & 171 \\
\hline 6 & - & 199 & 201 & - & 133 & 235 & 152 & 228 \\
\hline MW & 51 & 176.8 & 196.3 & - & 175.3 & 185.8 & 244.8 & 205.2 \\
\hline SD & 6 & 83.3 & 73.6 & - & 70.4 & 52.8 & 100.8 & 44.6 \\
\hline
\end{tabular}

cent premotor areas included areas beyond the precentral sulcus or close to the superior frontal sulcus of the contralateral hemisphere in two of the subjects.

We did, however, observe a left hemispheric predominance within the mesial frontal cortex: in five of six subjects there was activation above the left cingulate sulcus during (contralateral) right-hand movements, in three subjects during (ipsilateral) left-hand movements. Similar areas above the right cingulate sulcus became only activated in two subjects during (contralateral) left-hand movements (Table 2).

During the two bimanual tasks we observed two differential activation patterns: during the in-phase condition the number of active voxels in both lateral hemispheres were similar (Table 1). Right dorsolateral premotor areas anterior to the precentral gyrus in front of the primary motor hand area or close to the superior frontal sulcus were active in two subjects, left dorsolateral premotor areas in three subjects. During the anti-phase condition we observed a significant increase in right hemispheric activity. Two further subjects showed activity in the right dorsolateral premotor cortex. In addition, the number of significantly activated voxels increased in five of the six subjects during the anti-phase condition compared to the in-phase condition (Table 1; one-tailed paired $t$-test, $n=6, P<0.05$ ).

Within the mesial frontal cortex, the site of maximal activity was again in the areas close to and just above the left cingulate sulcus: they became activated in four of the six subjects during the in-phase condition and in all six during the anti-phase condition (Table 2). Within the right mesial cortex, areas above the right cingulate sulcus were only active in two subjects during the in-phase condition, but in five during the anti-phase condition, indicating also a clear increase in right hemispheric midline activity in the second condition. The direct comparison between the two bimanual conditions confirms that changes in medial premotor activity were not confined to the right hemisphere: in five of the six subjects there was also a significant increase in activation close to the left cingulate sulci (Table 2).

Modified region of interest analysis for medial wall areas

Attributing the foci of activity to the three predefined areas within the cortical medial wall allows the characterization of the relative shifts between the four conditions 
Table 2 SMA and anterior cingulate activity in individual subjects. Number of significant voxels in SMA and cingulate areas in the six individual subjects within the medial wall areas. Statistical significance levels: $P<0.001$, corrected for spatial extent $P<0.05$ for the first four comparisons (active conditions versus control); $P<0.01$ for the fifth comparison (anti-phase versus in-phase movements)

Table 3 Foci of activity within the medial wall areas. The number of " $x$ "s equals the number of the subjects with a significant activation within the described areas. The size of " $x$ " represents the degree of significance ( $t$-values) in the individuals $(x 2.33 \leq t<3.6 ; X$ $3.6 \leq t<5.0 ; X t \geq 5.0)$

\begin{tabular}{llllll}
\hline Subject & $\begin{array}{l}\text { Right hand } \\
P<0.001\end{array}$ & $\begin{array}{l}\text { Left hand } \\
P<0.001\end{array}$ & $\begin{array}{l}\text { In-phase } \\
P<0.001\end{array}$ & $\begin{array}{l}\text { Anti-phase } \\
P<0.001\end{array}$ & $\begin{array}{l}\text { Anti- vs in-phase } \\
P<0.01\end{array}$ \\
\hline 1 & 58 & - & 43 & 120 & 23 \\
2 & 37 & 24 & 50 & 110 & 16 \\
3 & 21 & 34 & - & 104 & 55 \\
4 & - & - & 51 & 99 & 5 \\
5 & 34 & - & 39 & 102 & 3 \\
6 & 47 & 66 & - & 65 & 2 \\
MW & 39.4 & 41.3 & 45.7 & 100 & 17.3 \\
SD & 12.5 & 17.9 & 5.0 & 17.1 & 18.5 \\
\hline
\end{tabular}

\begin{tabular}{lcc}
\hline & Right hemisphere & Left hemisphere \\
\hline Right-hand movements & & \\
$\begin{array}{l}\text { Dorsal SMA } \\
\text { Medial ant. cing./ventral SMA }\end{array}$ & $\mathrm{XXXX}$ \\
Lateral cingulate & $\mathrm{X}$ & $\mathrm{XXX}$ \\
Left-hand movements & $\mathrm{XXXX}$ \\
$\begin{array}{l}\text { Dorsal SMA } \\
\text { Medial ant. cing./ventral SMA }\end{array}$ & $\mathrm{XXXX}$ & $\mathrm{XXX}$ \\
Lateral cingulate & $\mathrm{XXXX}$ & $\mathrm{XXXX}$ \\
$\begin{array}{l}\text { In-phase movements } \\
\text { Dorsal SMA }\end{array}$ & $\mathrm{X}$ & $\mathrm{XX}$ \\
$\begin{array}{l}\text { Medial ant. cing./ventral SMA } \\
\text { Lateral cingulate }\end{array}$ & $\mathrm{XX}$ & $\mathrm{XXXXX}$ \\
Anti-phase movements & $\mathrm{XXXX}$ & $\mathrm{XXXXX}$ \\
$\begin{array}{l}\text { Dorsal SMA } \\
\text { Medial ant. cing./ventral SMA }\end{array}$ & $\mathrm{XXXXX}$ & \\
Lateral cingulate & $\mathrm{XX}$ & $\mathrm{XXX}$ \\
& $\mathrm{XXXXXX}$ & $\mathrm{XXXXXX}$ \\
& & $\mathrm{XXXXXX}$ \\
\hline
\end{tabular}

in the group of six subjects in more detail (Table 3). For unimanual movements, the results confirmed a predominantly left hemispheric activation pattern during righthand movement and a bilateral activation pattern during left-hand movements. There are, however, significant additional foci of activity within the lateral cingulate cortex, which occur predominantly within the hemispheres contralateral to the movements. Compared to the "singlesubject" results, some of them formed part of larger foci described above; others were, although highly significant locally, altogether too small to "survive" the statistical correction for spatial extent performed above.

The distribution of activity foci during bimanual movements showed clear differences between the three predefined areas. Within the dorsal SMA, left hemispheric activity was predominant during in-phase movements, and right and left hemispheric dorsal SMAs were active in the anti-phase condition. The medial anterior cingulate/ventral SMA areas were predominantly active on the left side during both in-phase and anti-phase movements. Lateral cingulate areas, on the other hand, showed similar degrees of activity on both hemispheres and again became more active during the anti-phase than the in-phase condition.

Between subjects, the exact site of maximal activation varied considerably within the mesial frontal cortex. Within individual subjects, however, in-phase movements showed a remarkable constancy of the activated sites compared with those activated during unimanual movements of the right and left hand. Activity during anti-phase movements, in contrast, showed a much wider distribution of activated areas including shifts of the maxima of activity and additional activity in mesial frontal and neighboring premotor areas.

\section{Discussion}

\section{Methodological considerations}

In this study we supplemented the group analysis by single-subject analysis for the evaluation of medial wall activation in uni- and bimanual movements. The progression from a group analysis to single-subject analysis, and ultimately to a modified region of interest analysis with a relaxed statistical threshold, revealed progressively more details about the diversity and exact site of the cortical activity. While the left medial wall dominance for unimanual right-hand movements was a common result for all three methods of statistical analysis, a bilateral involvement of SMA and medial cingulate areas during unimanual left-hand movements was not obvious from the group result, but demanded a single-subject analysis. A similar observation was made earlier for unilateral finger movement sequences (Schlaug et al. 1994). Cingulate involvement in the depths of the cingulate sulci con- 
tralateral to the unimanual movements only became apparent during the modified region of interest analysis. This last result exemplifies the importance of the selection of filter size (van Horn et al. 1998) and the benefits of lowering the level of statistical significance. While strong, local activity will survive conservative statistical thresholding, distributed activity might "get lost" when rigorous extent thresholds are applied (for discussion see Friston et al. 1996). On the other hand, a lower statistical threshold without spatial filtering increases the danger of "interpreting" artifacts. In these cases, the degree of reproducibility in different subjects provides an additional "safety net." We therefore chose to report the changes obtained during the modified region of interest analysis always for the whole group of subjects. The regions were defined according to the location of the clusters of activity foci. The definitions given in the "Materials and methods" are guided by the results of the imaging study and therefore do not necessarily follow anatomical borders.

\section{Dorsal medial wall areas}

The foci of activity within the dorsal medial wall were all above the cingulate or paracingulate sulci. With regard to the Talairach coordinate system, they were located close to and up to $3 \mathrm{~cm}$ behind the vertical line through the anterior commissure (AC line). This area presumably corresponds to the SMA proper (Zilles et al. 1996; Picard and Strick 1996). However, as more anterior parts of this area can be involved in more complex aspects of movement control, such as motor imagery (Stephan et al. 1995; Grafton et al. 1996), either a further subdivision of the SMA proper or a gradual change of neuronal properties from caudal to rostral SMA towards the more anterior pre-SMA characteristics (Luppino et al. 1991) is likely.

Initiation of movements (e.g., Thaler et al. 1988) and relay of sensory information for feedforward planning of movement sequences (Tanji and Shima 1994; Gerloff et al. 1997) have been suggested as important functions of SMA. Both occurred repeatedly during self-paced unimanual movements when subjects had to monitor their own performance rate, although somatosensory feedback may not be of major importance during repetitive tapping once the rhythm has been established (Ivry and Keele 1989). During simple right-hand index-finger movements, foci of SMA activity were observed mainly contralateral to the movements, which is in line with previous studies (Seitz et al. 1996; Kawashima et al. 1998). In contrast, left-hand movements led to bilateral SMA activity. Such greater recruitment of ipsilateral cortical motor areas during movements of the "non-dominant" compared to the "dominant" hand has been observed previously and may reflect the less "automatic" performance of the non-dominant hand (Mattay et al. 1998).

Bimanual in-phase movements were associated with similar or slightly increased degrees of activity within the left SMA compared to unimanual movements, and with a decrease in activity within the right SMA compared to left-hand movements alone. Compared to unimanual performance, bimanual in-phase movements included the additional task to initiate simultaneously finger movements of both hands. In most of our subjects, the left SMA was preferentially involved in this task. The right SMA became involved again, as soon as subjects perform anti-phase movements and therefore had to control their left hand independently from their right hand. These results suggest that initiation of movements generally involves the contralateral, and sometimes additionally the ipsilateral, SMA. This was true except for the case when both hands were moved simultaneously in the same direction. Then activity within the SMA of the dominant hemisphere seems to be sufficient to control both processes.

Ventral medial wall areas

While foci in the dorsal medial wall will correspond to SMA activity, it is much more difficult to decide whether the foci at the opening of the cingulate sulci and just above still represent activity within a ventral part of the SMA or are already within cingulate motor areas. The situation is further complicated by the anatomical variability in this area (Ono et al. 1990). In four of our six subjects, there was a prominent second cingulate sulcus or paracingulate sulcus (Paus et al. 1996) on the left side and in one also on the right. At least small parts of such sulci parallel to the cingulate sulci were also found in the other hemispheres. In a recent review, Picard and Strick (1996) reported a clustering of positron emission tomography (PET) activation sites within a posterior rostral cingulate zone close to the AC line, which might tentatively include the present site of activity. However, as cytoarchitectonic data are not yet available for a greater number of human subjects, we do not know where the average border between the supplementary motor area and the cingulate motor areas is situated and can therefore not decide with certainty whether the observed foci represent activity within cingulate motor areas or ventral SMA.

The foci of activity at the medial surface of the cingulate sulci were more prominent on the left than on the right hemispheres, regardless of whether the subjects moved their right hand, their left hand, or both. A left hemispheric predominance for processing of sequential aspects of motor programming and recognition of temporal patterns has been reported in healthy subjects (Gates and Bradshaw 1977; Peters 1985) and in patients (Mavlov 1980), suggesting that these ventral medial wall areas are included in hemispheric-specific aspects of movement control. Halsband et al. (1993) showed that deficits in rhythm reproduction from memory were more pronounced in patients with left premotor and SMA lesions than in those with right hemispheric premotor lesions. This was true for unimanual rhythm production of both the contralateral and the ipsilateral hand. In con- 
trast, patients with right-sided PMC lesions revealed a significant disturbance of rhythm reproduction for their contralateral hand only and when the rhythms had to be reproduced using both hands in an alternating manner (Halsband et al. 1993). This last observation is of special interest when compared with our imaging results: the right lateral hemisphere including premotor areas and right ventral medial wall areas became especially involved when subjects performed alternating, bimanual anti-phase movements, and only to a lesser extent during in-phase movements.

Areas in the depths of the cingulate sulci

Activity foci in the depths of the cingulate sulci were observed behind the vertical line through the anterior commissure (AC line) and presumably present activations within cingulate motor areas (CMA). The present resolution of the scans does not allow the determination of whether the foci were within the dorsal or ventral bank of the cingulate sulci. Caudal cingulate motor areas, close to or directly posterior to the AC line, become activated in most motor paradigms (Picard and Strick 1996).

In the present study, these cingulate foci of activity were predominantly contralateral to the movements (Table 3 ), which was not the case for the other medial wall areas (see above). Activity changes within cingulate sulcal areas were thus similar to those observed in primary motor areas. Movement-related activity in caudal cingulate cortex cells in monkeys (Shima et al. 1991), close anatomical connections between cingulate motor areas and primary motor cortex again in monkeys (Muakkassa and Strick 1979; Dum and Strick 1991) and a covariation of rCBF levels in cingulate sulcal areas and primary sensorimotor area during force control in humans (Dettmers et al. 1995) support the notion that caudal cingulate areas are involved in elementary processes of movement control in monkeys and humans. Why, then, did we not observe a further increase in cingulate activity during bimanual in-phase movements over and above that which would be expected from the combined unimanual conditions, even though the actual movement execution now has to be coordinated between both sides? Presumably, the increased coordinative effort is balanced by the reduced effort required to control motor variability, as such bilateral movements are characterized by a reduced timing variability compared to unimanual movements (Helmuth and Ivry 1996). As soon, however, as the more complex and less stable anti-phase movements are performed (Scholz and Kelso 1989, 1990; Byblow et al. 1994), this "advantage" is lost and a relative increase in activity is observed in cingulate motor areas of both sides.

\section{Medial wall areas and bimanual coordination}

The present results indicate that the different foci within medial wall areas represent activity within functionally distinct cingulate motor areas or supplementary motor areas. All of them became activated to varying degrees during bimanual and unimanual movements. This would suggest that they are not specific for bimanual coordination, but represent parts of a wider motor network, which can be involved during both uni- and bimanual movements (see also Kazennikov 1998, 1999). Nevertheless, their anatomical location close to the midline may predetermine them to control some of those aspects of movements which are especially important for the coordination of both hands. Based on kinematic recordings in patients with anterior cingulate lesions below the cingulate sulcus, we recently suggested that the integrity of cingulate areas and/or their connections are important to ensure unimanual independence and to facilitate exact temporal coordination between both hands (Stephan et al. 1999). Results of lesion studies may thus help to disentangle the significance of particular nodal points relative to the other components of the network.

Acknowledgements We thank our volunteers for their participation in these studies. The work was supported by the Deutscheforschungsgemeinschaft (SFB 194). Part of the data were reported previously in abstract form (Neuroimage 5, S270).

\section{References}

Brinkman C (1984) Supplementary motor area of the monkey's cerebral cortex: short and long-term deficits after unilateral ablation and the effects of subsequent callosal section. J Neurosci 4:918-929

Brinkman C, Porter R (1979) Supplementary motor area in the monkey: activity of neurons during performance of a learned motor task. J Neurophysiol 42:681-709

Byblow WD, Carson RG, Goodman D (1994) Expression of asymmetries and anchoring in bimanual coordination. Hum Mov Sci 13:3-28

Dettmers C, Fink GR, Lemon RN, Stephan KM, Passingham RE, Silbersweig D, Holmes A, Ridding MC, Brooks DJ, Frackowiak RSJ (1995) Relation between cerebral activity and force in the motor areas of the human brain. J Neurophysiol 74:802-815

Dum RP, Strick PL (1991) The origins of corticospinal projections from the premotor areas in frontal lobe. J Neurosci 11:667-689

Friston KJ, Holmes AP, Worsley KJ, Poline J-B, Frith CD, Frackowiak RSJ (1995a) Statistical parametric maps in functional imaging: a general linear approach. Hum Brain Mapping 2:189-210

Friston KJ, Frith CD, Turner R, Frackowiak RSJ (1995b) Characterizing evoked hemodynamics with fMRI. Neuroimage $2: 157-162$

Friston KJ, Holmes A, Poline JB, Price CJ, Frith CD (1996) Detecting activations in PET and fMRI: levels of inference and power. Neuroimage 4:223-235

Gates A, Bradshaw JL (1977) The role of the cerebral hemispheres in music. Brain Lang 4:403-431

Gerloff C, Corwell B, Chen R, Hallett M, Cohen LG (1997) Stimulation over the human supplementary motor area interferes with the organization of future elements in complex motor sequences. Brain 120:1587-602

Grafton ST, Arbib MA, Fadiga L, Rizzolatti G (1996) Localization of grasp representation in humans by positron emission tomography. 2. Observation compared with imagination. Exp Brain Res 112:103-111

Halsband U, Ito N, Tanji J, Freund H-J (1993) The role of premotor cortex and the supplementary motor area in the temporal control of movement in man. Brain 116:243-266 
Helmuth LL, Ivry RB (1996) When two hands are better than one: reduced timing variability during bimanual movements. J Exp Psychol 22:278-293

Horn JD Van, Ellmore TM, Esposito G, Berman KF (1998) Mapping voxel-based statistical power on parametric images. Neuroimage 7:97-107

Ivry RB, Keele SW (1989) Timing functions of the cerebellum. J Cogn Neurosci 1:136-152

Kawashima R, Matsumura M, Sadato N, Naito E, Waki A, Nakamura S, Matsunami K, Fukuda H, Yonekura Y (1998) Regional cerebral blood flow changes in human brain related to ipsilateral and contralateral complex hand movements - a PET study. Eur J Neurosci 10:2254-2260

Kazennikov O, Wicki U, Corboz M, Hyland B, Palmeri A, Rouiller EM, Wiesendanger M (1994) Temporal structure of a bimanual goal-directed movement sequence in monkeys. Eur J Neurosci 6:203-210

Kazennikov O, Hyland B, Wicki U, Pering S, Rouiller EM, Wiesendanger M (1998) Effects of lesions in the mesial frontal cortex on bimanual coordination in monkeys. Neuroscience $85: 703-716$

Kazennikov O, Hyland B, Corboz M, Babalian A, Roullier EM, Wiesendanger M (1999) Neural activity of supplementary and primary motor areas in monkeys and its relation to bimanual and unimanual movement sequences. Neuroscience (in press)

Laplane D, Talairach J, Meininger V, Bancaud J, Orgogozo JM (1977) Clinical consequences of corticectomies involving the supplementary motor area in man. J Neurol Sci 34:301-314

Luppino G, Matelli M, Camarda RM, Gallese V, Rizzolatti G (1991) Multiple representations of body movements in mesial area 6 and the adjacent cingulate cortex: an intracortical microstimulation study in the macaque monkey. J Comp Neurol 311:463-482

Mattay VS, Callicott JH, Bertolino A, Santha AK, Van Horn JD, Tallent KA, Frank JA, Weinberger DR (1998) Hemispheric control of motor function: a whole brain echo planar fMRI study. Psychiatry Res 83:7-22

Mavlov L (1980) Amusia due to rhythm agnosia in a musician with left hemisphere damage: a nonauditory supramodal defect. Cortex 16:331-338

Muakkassa KF, Strick PL (1979) Frontal lobe inputs to primate motor cortex: evidence for four somatotopically organized 'premotor' areas. Brain Res 177:176-182

Ono M, Kubik S, Abernathey CD (1990) Atlas of the cerebral sulci. Thieme, New York

Paus T et al in Cerebral Lortex 1996; 6:207-214

Peters M (1985) Performance of a rubato-like task: when two things cannot be done at the same time. Music Percept $2: 471-482$

Picard N, Strick PL (1996) Motor areas of the medial wall: a review of their location and functional activation. Cereb Cortex 6:342-353

Rouiller EM, Babalian A, Kazennikov O, Moret V, Yu X-H, Wiesendanger M (1994) Transcallosal connections of the distal forelimb representations of the primary and supplementary motor cortical areas in macaque monkeys. Exp Brain Res 102:227-243

Schlaug G, Knorr U, Seitz RJ (1994) Inter-subject variability of cerebral activations in acquiring a motor skill: a study with positron emission tomography. Exp Brain Res 98:523-534

Scholz JP, Kelso JAS (1989) A quantitative approach to understanding the formation and change of coordinated movement patterns. J Mot Behav 21:122-144

Scholz JP, Kelso JAS (1990) Intentional switching between patterns of 'bimanual' coordination depends on the intrinsic dynamics of the patterns. J Mot Behav 22:98-124

Seitz RJ, Schlaug G, Knorr U, Steinmetz H, Tellmann L, Herzog H (1996) Neurophysiology of the human supplementary motor area. Positron emission tomography. In: Lüders HO (ed) Supplementary sensorimotor area. Advances in neurology, vol 70. Lippincott-Raven, New York, pp 167-175

Shima K, Aya K, Mushiake H, Inase M, Aizawa H, Tanji J (1991) Two movement-related foci in the primate cingulate cortex observed in signal-triggered and self-paced forelimb movements. J Neurophysiol 65:188-202

Stephan KM, Fink GR, Passingham RE, Silbersweig D, CeballosBaumann AO, Frith CD, Frackowiak RSJ (1995) Functional anatomy of the mental representation of upper extremity movements in healthy subjects. J Neurophysiol 73:373-386

Stephan KM, Binkofski F, Halsband U, Dohle C, Wunderlich G, Schnitzler A, Tass P, Posse S, Herzog H, Sturm V, Zilles K, Seitz RJ, Freund HJ (1999) The role of ventral medial wall motor areas in bimanual co-ordination. A combined lesion and activation study. Brain 122:351-368

Talairach J, Tournoux P (1988) Co-planar stereotaxic atlas of the human brain. Thieme, Stuttgart

Tanji J, Shima K (1994) Role for supplementary motor area cells in planning several movements ahead. Nature 371:413-416

Tanji J, Okano K, Sato KC (1988) Neuronal activity in cortical motor areas related to ipsilateral, contralateral, and bilateral digit movements of the monkey. J Neurophysiol 60:325-343

Thaler DE, Rolls ET, Passingham RE (1988) Neuronal activity of the supplementary motor area (SMA) during internally and externally triggered wrist movements. Neurosci Lett 93:264-269

Travis AM (1955) Neurological deficiencies following supplementary motor area lesions in Macaca mulatta. Brain 78:174-198

Wiesendanger M, Rouiller EM, Kazennikov O, Perrig S (1996) Is the supplementary motor area a bilaterally organized system? In: Lüders HO (ed) Supplementary sensorimotor area. Advances in neurology, vol 70. Lippincott-Raven, New York, pp $85-94$

Zilles K, Schlaug G, Geyer S, Luppino G, Matelli M, Qü M, Schleicher A, Schormann T (1996) Anatomy and transmitter receptors of the supplementary motor areas in the human and nonhuman primate brain. In: Lüders HO (ed) Supplementary sensorimotor area. Advances in neurology, vol 70. LippincottRaven, New York, pp 29-43 